\title{
Mineral trioxide aggregate pulpotomy: patient selection and perspectives
}

\section{Prasad K Musale' \\ Sneha S Kothare' \\ Abhishek S Soni ${ }^{2}$ \\ 'Department of Pedodontics and Preventive Dentistry, M.A. Rangoonwala College of Dental Sciences and Research Centre, Pune, Maharashtra, India; ${ }^{2}$ Vanilla Smiles Dental Clinic, Pune, Maharashtra, India}

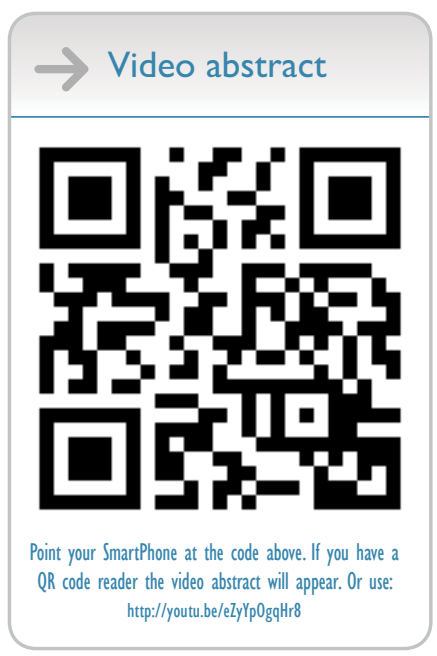

Correspondence: Prasad K Musale 3I, Gulmohar Garden, Lane 7, North Main Road, Koregaon Park, Pune 4l I00I, Maharashtra, India

Tel +9l 9822077597

Email pedoprass@gmail.com
This article was published in the following Dove Press journal: Clinical, Cosmetic and Investigational Dentistry

\begin{abstract}
This narrative aims at reviewing the available literature for mineral trioxide aggregate (MTA) pulpotomy to understand the procedure better and eventually improve the clinical and radiographic outcomes. An electronic search was conducted in PubMed, Cochrane, ScienceDirect and ClinicalKey databases with the following keywords: MTA pulpotomy, clinical outcomes, radiographic outcomes, primary teeth. No specific inclusion or exclusion criteria were applied as to what articles would be included in this review. The time period for the search began from 2001 with respect to MTA pulpotomy. However, this was not restrictive during the search. MTA pulpotomy has been a successful treatment modality in primary molars with proven success over
\end{abstract} the years. There is limited literature to support its success in primary incisors.

Keywords: MTA pulpotomy, primary teeth, deciduous teeth

\section{Introduction}

Dental caries remains one of the most prevalent health problems for children worldwide, especially in the developing countries. Management of deep carious lesions in the primary dentition is one of the biggest challenges faced by a pediatric dentist today. A large proportion of these lesions are associated with pulpal exposure due to the long-standing nature of the lesions. ${ }^{1,2}$ Several treatment options such as direct pulp capping, pulpotomy, pulpectomy and extraction may be considered depending on the extent of carious involvement and the experience of the dentist in managing such lesions. The outcome of any of these treatment modalities is largely dependent on the accurate assessment of the pulp status, the technique followed and the material used for the procedure.

Due to improved understanding of dental caries and advances in the available materials, the older concept of "extension for prevention" has been replaced by a more conservative approach toward management of dental caries. ${ }^{3}$ Deep carious lesions with pulpal exposure without any signs or symptoms of extensive pulpal degeneration are managed using pulpotomy procedure. Pulpotomy is defined as an endodontic procedure wherein the coronal pulp is amputated and the radicular healthy pulp is maintained within the root canals followed by placement of a medicament and good coronal seal until natural exfoliation of the primary tooth. Despite its proven clinical success over the years, the safety and effectiveness of the various pulpotomy agents have been questioned time and again. However, with the advent of newer biocompatible materials such as mineral trioxide aggregate (MTA), with proven success than the older agents, the future of this technique appears promising. ${ }^{4}$ 
Hence, the following review aims to compile the available literature on MTA pulpotomy from patient selection to clinical perspectives.

\section{Review}

\section{History of MTA pulpotomy}

The aim of pulpotomy is to preserve the radicular pulp, avoiding pain and swelling, and ultimately retain the tooth until natural exfoliation, thus preserving arch integrity. Formocresol has long been the gold standard medicament used for this procedure since its introduction by Sweet in 1932. Several authors have proven its success compared to other agents over the past 70 years. ${ }^{5}$ However, studies have shown that the success rates with formocresol decreased as the follow-up periods increased. The reported concerns of toxicity, mutagenicity and carcinogenicity further questioned its use. ${ }^{6}$ Thus, there was a need for a more biocompatible, nontoxic medicament. Hydraulic dental cements, with their more effective biocompatibility and proven success, were recommended for use as a pulpotomy agent. MTA, a hydraulic dental cement derived from Portland cement, was first introduced by Torabinejad et al at the Loma Linda University in the $1990 \mathrm{~s}^{7}$ and its first reported use was by Lee et al in $1993 .{ }^{8}$ As a pulpotomy agent, a preliminary report compared MTA with formocresol in 2001, wherein the teeth treated with MTA showed comparable clinical results with formocresol and higher incidence of pulp canal obliteration (PCO) after 17 months. ${ }^{9}$ Several studies that followed have proven the clinical and radiographic success of MTA as a pulpotomy material. ${ }^{10-12}$ Today, MTA pulpotomy is advocated for both primary molars as well as incisors. Further, the exposure could be either carious or iatrogenic. ${ }^{13}$

\section{MTA pulpotomy in primary molars}

MTA pulpotomy is indicated in primary molars with either a carious or iatrogenic exposure, after accurate preoperative clinical and radiographic evaluation of the tooth being considered. ${ }^{4}$ The tooth/teeth selected for the pulpotomy procedure should fulfill the criteria shown in the sections that follow.

\section{Clinical criteria}

1. Deep carious lesion with restorable crown: Carious lesions, especially proximal lesions, involving more than half the dentine thickness respond better to a pulpotomy approach than direct pulp capping due to their more extensive inflammatory reaction. The occlusal lesions showing distinct cavitation with visible dentin or extensive cavities involving more than half the occlusal surface (Interna- tional Caries Detection and Assessment System score 5 or 6) should be treated with pulpotomy procedure. ${ }^{13,14}$ This ensures the removal of inflamed coronal pulp tissue, leaving vital and uninflamed radicular tissue, which would have the potential for healing and repair. ${ }^{1}$ The extent of carious involvement is best observed with a radiographic examination. There should be adequate remaining tooth structure for proper retention of the definitive stainless steel crown (SSC) for adequate coronal seal. ${ }^{13,15}$ Additionally, the remaining tooth structure is necessary for rubber dam placement since the tooth should be considered non-restorable and is indicated for extraction if good isolation cannot be achieved. ${ }^{4}$

2. No history of systemic diseases or allergies: ${ }^{16}$ This is to eliminate the possibility of any underlying systemic pathology or allergy from affecting the final outcome of the procedure. ${ }^{17}$

3. No obvious signs of pulpal degeneration: History of spontaneous pain, sinus tract, pain evoked at night, pathologic mobility, and tenderness on percussion would warrant the tooth unsuitable for pulpotomy. ${ }^{15}$ Spontaneous pain and nocturnal pain are classical signs of irreversible pulpitis with extensive pulpal involvement. Presence of a fistula or sinus tract and/or mobility indicates spread of infection into the periradicular tissues. ${ }^{13,18}$

4. Carious or iatrogenic pulpal exposure: Vital pulp therapy is indicated in teeth with carious, mechanical/iatrogenic or traumatic exposures of pulp. ${ }^{4,15}$ Razlan and Wetzel (2006) opined that there is a difference in the pulpal response posttreatment based on the etiology of the pulpal exposure. ${ }^{19}$ However, Çelik and Sari negated this theory when they observed $100 \%$ success rates with both carious and mechanical exposures at the end of 18 months, although the rate of PCO was higher in case of carious exposure than in mechanically exposed teeth. ${ }^{13}$

5. Successful hemorrhage control within 5 min: Aeinehchi et al have stated that the arrest of bleeding should occur within $3 \mathrm{~min},{ }^{20}$ while Ozdemir et al have stated that the time elapsed should not be more than 5 min. ${ }^{21}$ However, Cohenca et al have suggested that the time period should not be more than 1-2 min. ${ }^{22}$ Failure of hemostasis after the application of a moistened cotton pellet within this time period indicates irreversible pulpitis and infection of the radicular pulp that warrants nonvital pulp therapy.

6. Color of hemorrhage: Bright red color of the intracanal hemorrhage is indicative of inflamed pulp, whereas dull red or brownish-red color of bleeding indicates extensive infection and pulpal degeneration. Pulpotomy should be performed when the intracanal hemorrhage is bright red 
in color. This color difference is due to the breakdown of the RBCs into hemosiderin by the inflammatory cytotoxic reactions. $^{13}$

\section{Radiographic criteria}

1. Remaining dentin thickness: The thickness of the remaining dentin in a deep carious lesion (International Caries Detection and Assessment System score 5 or 6) is best adjudged using a quality bitewing radiograph, which would aid a clinician ascertain the appropriate treatment protocol.

2. Physiologic root resorption more than one-third of the total root length: When the remaining physiologic root length is less than two-thirds of its entirety, vital pulp therapy is an unwarranted procedure as the tooth would exfoliate in the near future and performing the procedure would be unnecessary. ${ }^{15,18}$

3. Widened lamina dura: Radiographic widening of the lamina dura surrounding the roots is indicative of irreversible changes in the supporting tissues of the tooth root system warranting nonvital pulp therapy instead of vital pulp therapy. ${ }^{13}$

4. Presence of periradicular or furcal radiolucency: In primary teeth, the first radiographic signs of irreversible pulpal degeneration involving the radicular pulp are seen in the interfurcal region. Periradicular radiolucency in primary or permanent teeth results due to bacterial invasion of the supporting structures of the tooth. Diffuse radiolucency in these regions indicates more invasive endodontic therapy. ${ }^{13,23}$

5. Pathologic root resorption: Any external or internal root resorption evident in the radiographs of the concerned tooth would be indicative of inflammatory changes in the root canal system with infection of the radicular pulp. Such changes when witnessed on the radiographs are contraindicated for vital pulp therapy. ${ }^{13}$

6. Previously treated primary molars: According to Maroto et al, primary molars which have been previously treated should not be considered for pulpotomy. ${ }^{24}$ Teeth with failure of such a prior intervention are indicated for pulpectomy and not pulpotomy.

7. Primary teeth without a permanent successor: Musale and Soni stated that primary teeth with agenesis of the permanent successor are contraindicated for pulpotomy. ${ }^{16}$ The reason for this is that the required survival of the primary tooth is longer, and thus, performing pulpectomy with gutta-percha or MTA is preferable. ${ }^{25}$

\section{Technique}

Once a decision has been made, based on clinical and radiographic evaluation, to perform MTA pulpotomy in a particular tooth, a prior written informed consent should be obtained from the parent/guardian before initiating the procedure. After the informed consent is obtained, local anesthesia is administered prior to isolation. Hereafter, the procedure to be followed, as described by Maroto et al, is as follows:

1. Complete isolation with rubber dam: As stated previously, any endodontic procedure should be carried out under strict isolation using rubber dam, and in cases where this is not possible, pulpotomy should not be performed. ${ }^{15}$ The choice of isolation technique, that is, quadrant or single tooth isolation, is subjective in nature. However, quadrant isolation is preferred, if attainable, to facilitate crown preparation under rubber dam. ${ }^{17}$

2. Preparation of the molar and fitting of an SSC: Several authors have described crown preparation of a pulpotomized tooth at a later stage. However, crown preparation should precede the endodontic procedure as this would minimize the tooth structure loss and would, inevitably, be performed under local anesthesia, minimizing discomfort for the patient. Additionally, the crown fitting is done under rubber dam isolation, as has been recommended. ${ }^{16,17}$

3. Removal of the carious tissue with a round bur: Excavation of dental caries is initiated with a large, slow-speed, round bur after determining the extent of the lesion before pulpal exposure. ${ }^{16}$

4. Elimination of the roof of the pulp chamber and removal of the coronal pulp tissue: Deroofing of the pulp chamber is done with either a large, low-speed, round bur or a \# 330 carbide bur. Some authors have even suggested the use of a safe-end/non-cutting end taper fissure bur for the same. ${ }^{15,16}$ After the deroofing is complete, the coronal pulp is amputated. This is done using either a large, low-speed, round bur (\# 6 or \# 8 round bur) (5-27 $^{25}$ or a sharp spoon excavator. ${ }^{15,28}$

5. Disinfection of the pulp chamber: Some studies recommend disinfection of the pulp chamber with 3\%-5\% sodium hypochlorite $(\mathrm{NaOCl})$ solution prior to drying of the chamber. This is known to dissolve and remove any remnant tissue, debris or dentinal chips which can lead to infection of the radicular pulp. ${ }^{7}$ Akcay et al compared the effects of using $5 \% \mathrm{NaOCl}$ and physiologic saline for disinfection of the pulp chamber before placement of pulpotomy medicament in the teeth, which showed successful treatment outcomes with MTA. Of the two groups, on histologic evaluation, it was seen that only one of eight teeth in the saline group showed any inflammation, and this was minimal. All eight teeth had hard tissue bridge formation, including seven complete and 
one incomplete bridge (one with an atubular structure and the remainder with irregular tubular structure); seven of the eight teeth showed odontoblastic layer continuity and only one of eight teeth showed internal resorption. In the $5 \% \mathrm{NaOCl}$ group, inflammation was not observed in any of the teeth. All nine teeth had complete bridge formation (with regular or irregular tubular structure); eight of nine teeth showed odontoblastic layer continuity and one of nine teeth showed internal resorption, but the resorptive area appeared to be filled with reparative dentin at the end of the follow-up period. Thus, the cleansing action of $\mathrm{NaOCl}$ is non-contributory to the outcome of pulpotomy procedure. ${ }^{29}$ Irrigation of the pulp chamber using normal saline is adequate to remove any debris remaining in the chamber.

6. Drying and control of the pulp hemorrhage using slight pressure with a sterile cotton wool pellet: As stated previously, the control of bleeding should be attained within $3-5 \min ^{18}$ using a moist, sterile, cotton wool pellet. Gentle pressure should be applied against the canal orifices with this pellet. A moistened cotton pellet is recommended since the fibers from a dry cotton pellet would get incorporated in the clot and be dislodged when the pellet is removed and thus hemorrhage will resume. At the end of the above stipulated time, on removal of the pellet, the pulp chamber should be devoid of any remnant coronal pulp tissue and hemostasis should be attained at the canal orifices. If at this stage hemorrhage still persists, one should check for remaining pulpal tissue tags and remove them immediately to attain hemostasis. ${ }^{23}$ To achieve this hemostasis, the use of a cotton pellet soaked with $1.25 \%-6 \% \mathrm{NaOCl}$ is recommended for $1 \mathrm{~min}^{7}$ The $\mathrm{NaOCl}$ would dissolve any extrapulpal blood clot present between the wound surface and the medicament that interferes with healing. Shröder suggested that the presence of an extrapulpal blood clot potentiated the initial inflammatory response triggered by a pulpotomy medicament due to the chemotactic effect of the polymorphonuclear leukocytes of the fibrin in the clot. The presence of such a clot could lead to internal resorption in the dentin adjacent to it rather than it being the effect of the medicament. ${ }^{30}$ If, after the removal of the moistened pellet, hemorrhage recommences, the radicular pulp is chronically inflamed, and the tooth is contraindicated for pulpotomy and pulpectomy procedure should be undertaken. ${ }^{28}$

7. Mixing of the MTA with sterile water on a glass slab following the manufacturer's instructions: The choice of type of MTA is subjective in nature. Cardoso-Silva et al compared gray MTA and white MTA as the pulpotomy agent. Higher dentin bridge formation was found with gray MTA, while more necrosis and inflammatory cells were seen with white MTA in histologic evaluation, although the overall success was similar with both the materials. This difference in the two materials was attributed to tetracalciumaluminoferrite content in the material. ${ }^{31}$ Kang et al, in a randomized controlled trial, found the clinical and radiographic success rates of ProRoot MTA, OrthoMTA and RetroMTA to be indistinguishable. ${ }^{32}$

MTA should be mixed with sterile water as per the manufacturer's recommendation on a clean sterile glass slab and a spatula. ${ }^{23}$ The final mix should have a wet, sand-like consistency. ${ }^{15}$

8. Pressing the MTA to the walls and floor of the pulp chamber with a cotton wool pellet moistened in sterile water: Once the MTA is mixed, the cotton pellet, placed within the pulp chamber, should be removed and the MTA mix carried into it using an excavator, a retrograde amalgam carrier ${ }^{23}$ or a Dovgan carrier. The increments of MTA should be compacted against the floor and walls of the chamber using a cotton pellet moistened with sterile water. ${ }^{16}$ The thickness of the compacted bulk of material should be 3-4 mm and should cover all of the root canal orifices and the floor. ${ }^{23}$ No voids should be seen. It is advisable to make a radiograph at this stage to check for adequate thickness of the MTA layer and proper compaction of the material.

9. Filling the pulp chamber with a glass ionomer cement: After verifying the acceptability of the MTA layer, the remainder of the pulp chamber should be restored with glass ionomer cement immediately. ${ }^{16}$ This concept of immediate restoration with glass ionomer cement is supported by some authors, ${ }^{31}$ while others state that the MTA should be covered by a moist cotton pellet followed by temporization and the patient be recalled after $24 \mathrm{~h}$ to remove the pellet and fill the chamber with glass ionomer cement. ${ }^{13}$ However, this is not necessary with the newer MTA material available in the market that has a shorter setting time than traditional ProRoot MTA or Portland cement.

10. Cementation of the SSC using glass ionomer luting cement: After the pulp chamber is restored with glass ionomer cement, the predetermined and fitted SSC should be cemented with glass ionomer luting cement. Several other definitive restorations have been carried out in various studies, including amalgam restorations; however, SSCs have been found to be the most successful 
definitive restoration. Well-adapted SSCs eliminate the chances of procedural failure due to fracture of restoration or microleakage. ${ }^{4}$ After cementation, the excess glass ionomer cement should be removed and the proximal contacts be flossed using knotted floss. Randall has recommended the use of resin-modified glass ionomer cements for cementation of the crown. ${ }^{17}$

11. Oral hygiene instructions and regular follow-up: The patient and parent should be given proper oral hygiene maintenance instructions, since in most cases, discomfort or pain reported by the patient is due to lack of brushing that area. Patients should be asked to maintain the shiny metallic appearance of the SSC. ${ }^{17}$ Further, the patient should be followed up on a regular basis with recall scheduled every 6 months or as part of the individual patient's routine periodic examination. ${ }^{4}$

\section{Follow-up}

The pulpotomized tooth should be followed up regularly. On the recall visit, the tooth should be examined for any of the given signs and symptoms (Table 1).

Dentin bridge formation and PCO, also seen radiographically, are not considered a failure as they maintain the vitality of the radicular pulp ${ }^{21}$ and occur due to the increased transforming growth factor- $\beta 1$, BMP secretion and induction of osteodentine, along with the associated formation of Type I collagen and osteonectin with increased expression of dentin sialoprotein and nestin, respectively.
Reactive root resorption is considered a failure of the treatment. Smith et al reported that the resorptive processes are of two types - dental and osseous. Internal resorption is of dental origin and could be attributed to the pulpal response secondary to the medicament; it should be considered a success unless associated with any osseous change. Osseous resorption should be considered a failure as it is pathologic in nature. ${ }^{6}$

Depending on the clinical and radiographic outcomes, the periodicity of the subsequent evaluation should be determined (Table 2).

Several studies have considered the above general parameters of assessing the outcome of the procedure without due assessment of the outcome histologically. However, some of the recent randomized controlled trials followed the modified Zurn and Seale criteria (2008) which provide both clinical and radiographic scoring criteria to evaluate the tooth in the follow-up period. The advantage of using this scoring system is that it encompasses all the clinical and radiographic changes that are frequently witnessed with MTA pulpotomy, including the occurrence of MTA stabilization. ${ }^{16}$

Oral hygiene instructions and importance of oral hygiene maintenance should be reiterated to the patient and parent at all recall visits. ${ }^{17}$

\section{MTA pulpotomy in primary incisors}

Pulpotomy as a treatment protocol is not commonly associated with primary anterior teeth. Literature concerning

Table I MTA pulpotomy outcomes

\begin{tabular}{|c|c|c|}
\hline Outcomes & Successful & Failure \\
\hline \multirow[t]{7}{*}{ Clinical outcomes } & Asymptomatic & Long-lasting chewing sensitivity \\
\hline & Natural exfoliation & Spontaneous pain \\
\hline & Exfoliation prematurely due to ectopic eruption & Gingival swelling approximating the furcation area \\
\hline & Physiologic mobility & Periodontal pocket formation \\
\hline & Gingival inflammation due to poor oral hygiene & Pathologic mobility $>2 \mathrm{~mm}$ \\
\hline & Short-lasting chewing sensitivity & Sinus tract/fistula formation \\
\hline & & Premature tooth loss due to pathology \\
\hline Radiographic & Normal taper of root canals & Widened periodontal ligament space \\
\hline \multirow[t]{5}{*}{ outcomes } & Normal width of periodontal ligament space & Furcation radiolucency \\
\hline & No trabecular changes & External root resorption \\
\hline & Nonperforating internal resorption & Perforating internal resorption \\
\hline & Dentin bridge formation & Osseous radiolucency involving the permanent successor \\
\hline & Pulp canal obliteration & crypt \\
\hline Patient-oriented & Asymptomatic & Nocturnal pain \\
\hline \multirow[t]{5}{*}{ outcomes } & Short-lasting tenderness on chewing & Long-lasting tenderness on chewing \\
\hline & & Swelling - gingival or extraoral \\
\hline & & Purulent discharge \\
\hline & & Halitosis \\
\hline & & Marked mobility \\
\hline
\end{tabular}

Abbreviation: MTA, mineral trioxide aggregate. 
Table 2 Recall recommendations after MTA pulpotomy

\begin{tabular}{ll}
\hline Outcomes & Recall \\
\hline Asymptomatic & 6-month recall \\
Natural exfoliation & \\
Exfoliation prematurely due to ectopic eruption & \\
Normal taper of root canals & \\
Normal width of periodontal ligament space & \\
No trabecular changes & 3-month recall \\
\hline Physiologic mobility & \\
Gingival inflammation due to poor oral hygiene & \\
Short-lasting chewing sensitivity & \\
Nonperforating internal resorption & \\
Dentin bridge formation & \\
Pulp canal obliteration & \\
\hline Long-lasting chewing sensitivity & \\
Spontaneous pain & \\
Gingival swelling approximating the furcation area & \\
Widened periodontal ligament space & \\
Furcation radiolucency & \\
\hline Periodontal pocket formation & \\
Pathologic mobility $>2$ mm & \\
Sinus tract/fistula formation & \\
Premature tooth loss due to pathology & \\
External root resorption & \\
Perforating internal resorption & \\
Osseous radiolucency involving the permanent & \\
successor crypt & \\
\hline Abbrevion & \\
\hline
\end{tabular}

Abbreviation: MTA, mineral trioxide aggregate.

pulpotomy in primary incisors is scarce with none concerning MTA alone and only one study comparing ferric sulfatecombined MTA (FS+MTA) pulpotomy with pulpectomy. Nguyen et al found in this study that FS+MTA pulpotomy had comparable clinical and radiographic outcomes after 18 months, and suggested it as an alternative to zinc-oxide eugenol pulpectomy in carious asymptomatic primary maxillary incisors with carious exposure on excavation. ${ }^{33}$

\section{Conclusion}

MTA as a pulpotomy agent has shown favorable results in case of primary molars. There is limited literature available regarding its use in primary incisors. However, the success of vital pulp therapy with MTA depends on proper case selection and technique of management of the tooth than the material itself.

\section{Disclosure}

The authors report no conflicts of interest in this work.

\section{References}

1. Kassa D, Day P, High A, Duggal M. Histological comparison of pulpal inflammation in primary teeth with occlusal or proximal caries. Int $J$ Paediatr Dent. 2009;19(1):26-33.
2. Farsi N, Alamoudi N, Balto K, Mushayt A. Success of mineral trioxide aggregate in pulpotomized primary molars. $J$ Clin Pediatr Dent. 2005;29(4):307-311.

3. Frencken JE, Innes NP, Schwendicke F. Managing carious lesions: consensus recommendations on carious tissue removal. Adv Dent Res. 2016;28(2):46-48.

4. American Academy of Pediatric Dentistry. Pulp therapy for primary and immature permanent teeth. Pediatr Dent. 2017;39(6): 325-333.

5. Fuks A, Guelmann M, Kupietzky A. Current developments in pulp therapy for primary teeth. Endod Topics. 2012;23(1):50-72.

6. Smith NL, Seale NS, Nunn ME. Ferric sulfate pulpotomy in primary molars: a retrospective study, Pediatr Dent. 2000;22(3):192-199.

7. Dammaschke T, Camp JH, Bogen G. MTA in Vital Pulp Therapy. In: Torabinejad M, editor. Mineral Trioxide Aggregate: Properties and Clinical Applications. Danvers, MA: John Wiley \& Sons, Inc; 2014.

8. Lee SJ, Monsef M, Torabinejad M. Sealing ability of a mineral trioxide aggregate for repair of lateral root perforations. $J$ Endod. 1993;19(1);541-544.

9. Holan G, Eidelman E, Fuks AB, Long-term evaluation of pulpotomy in primary molars using mineral trioxide aggregate or formocresol. Pediatr Dent. 2005;27(2):129-136.

10. Naik S, Hegde AH. Mineral trioxide aggregate as a pulpotomy agent in primary molars: an in vivo study. J Indian Soc Pedod Prev Dent. 2005;23(1):13-16.

11. Niranjani K, Prasad MG, Vasa AA, Divya G, Thakur MS, Saujanya K. Clinical evaluation of success of primary teeth pulpotomy using mineral trioxide aggregate ${ }^{\mathbb{}}$, laser and biodentine ${ }^{\mathrm{TM}}$-an in vivo study. J Clin Diagn Res. 2015;9(4):ZC35-7.

12. Haghgoo R, Abbasi F. Clinical and radiographic success of pulpotomy with MTA in primary molars: 30 months follow up. Iran Endod $J$. 2010;5(4):157-160.

13. Çelik BN, Sari S. Carious exposure versus mechanical exposure for MTA pulpotomy in primary teeth. BioMed Res Int. 2016;2016:2753429.

14. Braga MM, Mendes FM, Ekstrand KR. Detection activity assessment and diagnosis of dental carious lesions. Dent Clin NorthAm. 2010;54(3):479-493.

15. Rodd HD, Waterhouse PJ, Fuks AB, Fayle SA, Moffat MA, UK National Clinical Guidelines in Pediatric Dentistry. Int J Paed Dent. 2006;16(Suppl 1):15-23.

16. Musale PK, Soni AS. Clinical pulpotomy trial of Copaifera langsdorffii oil resin versus formocresol and white mineral trioxide aggregate in primary teeth. Pediatr Dent. 2016;38(2):5-12.

17. Randall RC. Preformed metal crowns for primary and permanent molar teeth: review of the literature. Pediatr Dent. 2002;24(5):489-500.

18. Mielke CH Jr, Kaneshlro MM, Maher IA, Weiner JM, Rapaport SI. The standardized normal ivy bleeding time and its prolongation by aspirin. Blood. 1969;34(2):204-215.

19. Raslan N, Wetzel WE. Exposed human pulp caused by trauma and/or caries in primary dentition: a histological evaluation. Dent Traumatol, 2006;22(3):145-153.

20. Aeinehchi M, Dadvand S, Fayazi S, Bayat-Movahed S. Randomized controlled trial of mineral trioxide aggregate and formocresol for pulpotomy in primary molar teeth. Int Endod J. 2007;40(4): 261-267.

21. OzdemirY, Kutukculer N, Topaloglu-Ak A, Kose T, Eronat C. Comparative evaluation of pro-inflammatory cytokine levels in pulpotomized primary molars. J Oral Sci. 2015;57(2):145-150.

22. Cohenca N, Paranjpe A, Berg J. Vital pulp therapy. Dent Clin North Am. 2013;57(1):59-73.

23. Waterhouse PA, Whitworth JM, Camp JH, Fuks AB. Pediatric Endodontics: Endodontic Treatment for Primary and Young Permanent Dentition in Cohen's Pathways of Pulp. 10th ed. St Louis, MO: Elsevier Inc; 2011.

24. Maroto M, Barbería E, Vera V, García-Godoy F. Mineral trioxide aggregate as pulp dressing agent in pulpotomy treatment of primary molars: 42-month clinical study. Am J Dent. 2007;20(5):283-286. 
25. Tunc ES, Bayrak S. Usage of white mineral trioxide aggregate in a nonvital primary molar with no permanent successor. Aus Dent J. 2010;55(1): 92-95.

26. Strange DM, Seale NS, Nunn ME, Strange M. Outcome of formocresol/ ZOE sub-base pulpotomies utilizing alternative radiographic success criteria. Pediatr Dent. 2001;23(4):331-336.

27. Fernandez CC, Martinez SS, Jimino FG, Rodriguez AIL, Mercade M, Short-term treatment outcome of pulpotomies in primary molars using mineral trioxide aggregate and Biodentine: a randomized clinical trial. Clin Oral Investig. 2015;20(7):1639-1645.

28. Huth KC, Paschos E, Hajek-Al-Khatar N, et al. Effectiveness of 4 pulpotomy techniques-randomized controlled trial. JDent Res. 2005;84(12):1144-1148.

29. Akcay M, Sari S, Duruturk L, Gunhan O. Effects of sodium hypoclorite as disinfectant material previous to pulpotomies in primary teeth. Clin Oral Investig. 2015;19(4):803-811.
30. Shröder U. Effects of calcium hydroxide-containing pulp-capping agents on pulp cell migration, proliferation, and differentiation. J Dent Res. 1985;64(Spec Iss):541-548.

31. Cardoso-Silva C, Barberia E, Maroto M, Garcı-Godoy F. Clinical study of mineral trioxide aggregate in primary molars. Comparison between grey and white MTA-a long term follow-up (84 months). J Dent. 2011;39(2):187-193.

32. Kang CM, Kim SH, Shin Y, et al. A randomized controlled trial of ProRoot MTA, OrthoMTA and RetroMTA for pulpotomy in primary molars. Oral Dis. 2015;21(6):785-791.

33. Nguyen TD, Judd PL, Barrett EJ, Sidhu N, Casas MJ, Comparison of ferric sulfate combined mineral trioxide aggregate pulpotomy and zinc oxide eugenol pulpectomy of primary maxillary incisors: an 18-month randomized, controlled trial. Pediatr Dent. 2017;39(1):34-38.
Clinical, Cosmetic and Investigational Dentistry

\section{Publish your work in this journal}

Clinical, Cosmetic and Investigational Dentistry is an international, peer-reviewed, open access, online journal focusing on the latest clinical and experimental research in dentistry with specific emphasis on cosmetic interventions. Innovative developments in dental materials, techniques and devices that improve outcomes and patient satisfac-

\section{Dovepress}

tion and preference will be highlighted. The manuscript management system is completely online and includes a very quick and fair peerreview system, which is all easy to use. Visit http://www.dovepress. com/testimonials.php to read real quotes from published authors. 